\title{
Simulation of Aging Metal with Preservative Coating
}

\author{
Yutaro Kamata $^{1}$ Yoshitsugu Manabe $^{1}$ Noriko Yata $^{1}$
}

${ }^{1}$ Chiba University

\begin{abstract}
This paper describes a method of texture reproduction of realistic metal surfaces with age deterioration for applying in computer graphics. We propose a new simulation method for the peeling of preservative coatings of surfaces; the method considers the object geometry and surrounding environment. We also propose a method to decrease processing time using probability distribution maps to speed up the simulation.
\end{abstract}

Keywords: Texture, Simulation, Processing time, Geometric calculation.

\section{Introduction}

The reproduction of textures is an important factor in computer graphics (CG ). Texture mapping is the most popular method for the reproduc-tion of textures in current CG. This method enables addition of texture information for the surfaces of geometric objects by applying a texture image. Therefore, the quality of the reproduced texture depends on the realism of the applied texture image.

When various materials or conditions are reproduced with texture mapping, the age deterioration of surfaces is an important factor in achieving realistic texture repro-duction for CG objects.

For the reproduction of deterioration, age-deteriorated texture is very important; the texture is, however, difficult to manually generate. Therefore, a method to automatically generate agedeteriorated texture is required. Several simulation studies have been conducted for generating aging textures.

Metal texture is one of the most studied materials for realistic reproductions. However, because alloys such as zinc and preservative coatings such as benzotriazole are commonly used in metal products, the actual exposed metal surface of the deteriorated metal product is not often observed. Therefore, we consider that factors of deterioration are related to not only chemical but also physical aspects such as touching or striking of an object's surfaces. In this study, we focused on the effects of peeling off of preservative coatings and the corrosion that expands from the peeled area. We propose simulation of deteriorated metal with a preservative coating on an object surface for more realistic CG textures.

\section{Related work}

Many studies have examined the reproduction of deterioration. For example, N. Merillou et al. [1] studied the effects of weathering; Olivier Clement et al. [2] calculated the deterioration area by colorations. N. Merillou et al. focused on deterioration and material features [3]. Yao-Xun proposed simulation of deteriorated metal surfaces for the texture representation of old metallic products [4]. M. M. Stack proposed the simulation of metallic erosion and corrosion for realistically modeling deteriorated metal surfaces [5]. In these studies, the object is exposed, and the whole surface can be corroded by environmental effects. 
The distribution of age-deteriorated areas of surfaces is generally influenced by the geometric features of objects. Tien-Tsin Wong et al. reproduced an agedeteriorated texture with a simple simulation using geometric features to enhance the quality of rendered images [6]. Jianye $\mathrm{Lu}$ et al. considered the effects on geometric features by changing the type of deterioration or material surface [7]. They observed and analyzed the age deterioration of real objects.

Yao Xun et al. proposed simulation of aging in metals to reproduce corrosion caused by sea water [8]. Their results showed that considering the effects of moving metallic ions when calculating expanding corrosion improves the quality of the reproduction. Stephane Merillou et al. showed that the metal corrosion on an object's surface expands in the surrounding area and the depth direction [9]. The results of the reproduction of metal collapse in that study indicated that corrosion influences an object's texture and shape.

\section{Simulation of deterioration}

The proposed method for the simulation of deterioration consists of two steps. First, peeling of the preservative coating by striking the target surface with other objects such as water drops is calculated. Second, the subsequent corrosion and expansion the corrosion area on the object's surfaces are calculated. The two steps are related. Corrosion points in the second step are determined by the areas where the coating is peeled, which is calculated in the first step, and the durability of the preservative coating in the first step is reduced by the expansion of corrosion areas calculated in the second step.

\subsection{Calculation of peeling of preserv- ative coating}

First, objects that strike the surface of the metal object are generated. Pressure is applied to the metal object surface using the generated objects, which leads to a pressure value accumulation in the target area. The pressure value is calculated by Hertz's rule, expressed as

$$
P=\frac{3 N}{2 \pi a^{2}} \sqrt{1-\left(\frac{r}{a}\right)^{2}}
$$

where $P$ is the pressure value, $N$ is the weight of the generated object, $a$ is the area the generated object strikes, and $r$ is the radius of the generated object. When the accumulated pressure in the target area exceeds a threshold value, the preservative coating peels in this area. Itoh et al. have observed that corrosion underneath the coating changes the durability of the preservative coating and that corrosion expands from these corroded areas [10].

The durability of the preservative coating is expressed as

$$
P^{\prime}=P_{i}+\frac{P}{D^{\prime}},
$$

where $P i$ is the accumulated pressure in area $i$, and $D$ is the endurance of the preservative coating. If $\mathrm{Pi}$ exceeds a threshold value, the coating of area $i$ is peeled. In the simulation, if the preservative coating is a metal alloy coating such as zinc, area $i$ is chipped, and if it is a chemical coating such as benzotriazole, area $i$ is a unit area in texture space.

\subsection{Calculation of corrosion and ex- pansion of corrosion area}

The onset of corrosion is in the area where the preservative coating has been peeled. In the corrosion calculation, the 
tendency of corrosion $T i$ is influenced by the geometric features of the object's surface; $T i$ is expressed as

$$
T_{i}=V+O+R+S,
$$

where $V, O, R$, and $S$ are the geometric features, namely, convexity, occlusion, orientation, and position, respectively. The tendency determines the accumulated corrosion value $C i$ for each surface area as

$$
C^{\prime}=C_{i}+T_{i} C
$$

where $C$ is the volume of corrosion determined by the calculation. Corrosion expands to surrounding areas from an area in which the accumulated corrosion value exceeds the threshold value. For reproducing the corrosion phenomenon, the preservative coating is ignored while expanding corrosion and the corrosion value is increased.

The proposed method for the simulation of corrosion calculates the abovementioned steps to reproduce agedeteriorated metal surface texture.

\subsection{Simulation results}

The results of the simulation of deterioration are shown in Figures 1 and 2. Figure 1 shows a comparison of corrosion expansion simulation by a previous method that considered an object without a preservative coating and that by the proposed method, which considered a preservative coating. It can be seen that although similar initial corrosion conditions were considered, the result of the proposed method did not show corrosion of the entire surface of the object, unlike the simulation result of the previous method. Textures such as those generated by the proposed method are often seen on the surfaces of real objects. Figure 2 shows a comparison of different conditions for the aging simulation. These results were obtained after sufficient time had elapsed to allow the surfaces to deteriorate under each condition. The comparison between coated and noncoated surfaces shows that the deteriorated surface with the preservative coating is more similar to the deteriorated surface of an actual metal product. Moreover, the comparison between corrosion expansion and no corrosion shows that the surface with corrosion reproduces a more natural and realistic texture.

Thus, the proposed method is effective for reproducing age-deteriorated metal textures.
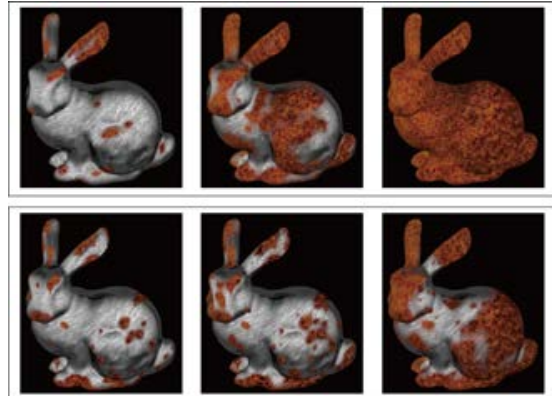

Fig. 1: Comparison of simulation of corrosion expansion area by a previous and proposed method. Simulation time goes from left to right. Top is exposed surface and bottom is surface with preservative coating.

\section{Reducing processing time using maps}

Typically, a fundamental tradeoff exists between quality and processing time; higher quality requires greater processing time. Therefore, we present a method to simulate texture generation with minimum processing time while maintaining a high level of accuracy.

To decrease the processing time required for texture simulation, we propose a simulation in a two-dimensional texture space considering geometric and environmental feature maps, as shown in Figure 3. 


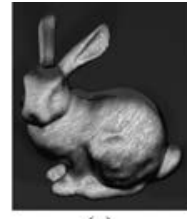

(a)

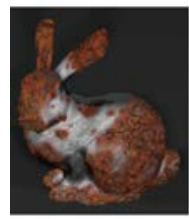

(d)

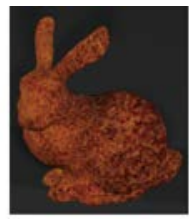

(b)

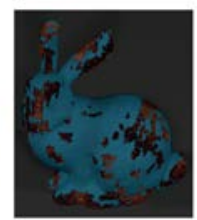

(e)

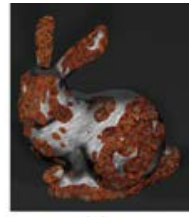

(c)
Fig. 2: Results of the deteriorative simulation: (a) original, (b) exposed surface, (c) nonexpanding corrosion area with chemical preservative coating, (d) expanding corrosion area with chemical preservative coating, and (e) metal preservative coating.

\subsection{Geometric maps}

The geometric maps are constructed from the geometric features of each object, such as convexity, occlusion, orientation, and position. The amount of pressure generated by collisions was calculated by approximating a convex distribution, as shown in Figure 4.

There is a correlative relationship between the geometric map and distribution of physical factors of deterioration. Figure 5 shows that the geometric map of the Stanford Bunny using convexity and occlusion values is similar to a distribution of the striking points obtained from 50,000 simulated collisions using small to large objects. Therefore, the calculation of striking pressure can be simplified, and the number of strikes can be reduced by considering that object surfaces are joined in two-dimensional space and by correcting pressure expansion using geometric maps.

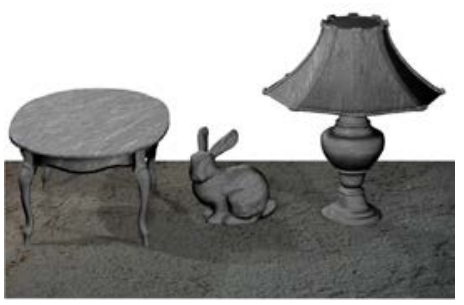

(a)

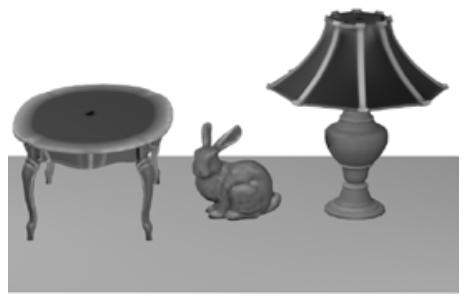

(b)

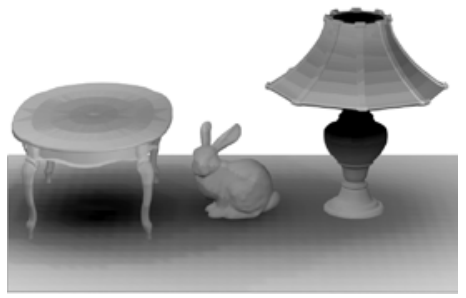

(c)

Fig. 3: Feature maps for texture simulation(brightness reproduces each map's values) (a) original, (b) geometric map, and (c) Environmental map

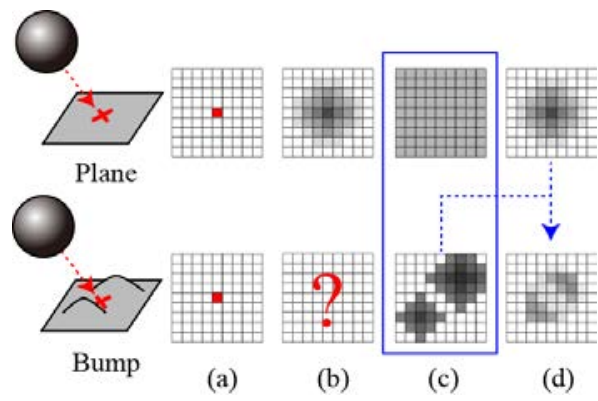

Fig. 4: Determining the pressure value: (a) contact point in two-dimensional space, (b) pressure distribution, (c) roughness map, and (d) pressure distribution using roughness map 

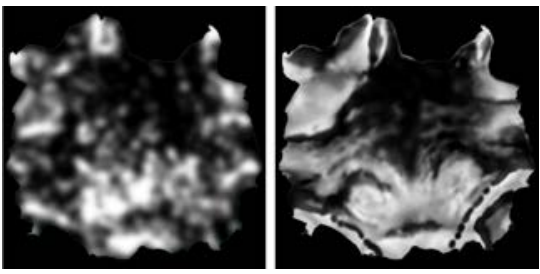

Fig. 5: Correlative relationship between a geometric map and a distribution of hit points on the surfaces of the Stanford Bunny. Left is the distribution of striking point and right is the geometric map.

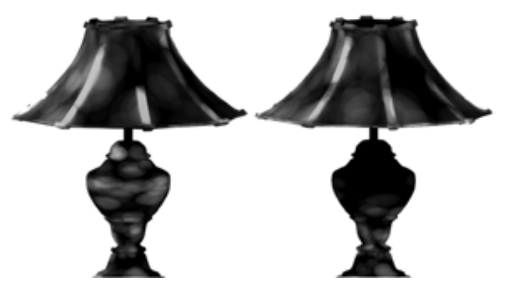

Fig. 6: Comparison of the predicted striking points on the surfaces of a lamp and the shade with and without an environmental map. Left is the distribution of striking point without environmental map and right is using it.

\subsection{Environmental maps}

The environmental maps are constructed by measuring the distances and angles between each target object and surrounding objects. To use the proposed method in the simulation of deterioration, surfaces are shaped by touching other objects over an assumed period of time in a particle simulation, and the striking points on the surfaces are predicted by approximating the probability distribution.

For example, the striking points on the surfaces of a lamp and a shade are predicted by approximating the probability distribution, as shown in Figure 6. In this figure, prediction by probability distribution is effective for the threedimensional collision simulation because there are no points inside the lampshade.
Thus, simulation in a threedimensional space can be simplified using the environmental map. In addition, the processing time required for iterative calculations for the simulation of aging deterioration can be significantly reduced.

\section{Texture reproduction and pro- cessing time measurement}

The reproduction of a deteriorated metal texture using the proposed methods is shown in Figure 7. In this figure, there are six types of objects: the Stanford Bunny, a lamp, a lampshade, a table board, a table side, and table feet. The processing time required for generating each texture is shown in Table 1.

Generally, for the effective reproduction of realistically aged metal surfaces, the following should be considered when generating textures.

- The peeled coating and corroded area should be reproduced in a convex form with few occlusion areas.

- The peeled area expanded to surrounding areas should be reproduced.

- The corroded area that proceeds underneath the preservative coating should also be reproduced.

The proposed methods reduce the simulation processing time significantly and reproduce high quality textures that satisfy the abovementioned considerations for reproducing realistically aged metal surfaces.

Table 1: The comparison of the processing time to make textures in Figure 7

\begin{tabular}{|c|c|c|}
\hline $\begin{array}{l}\text { Simulation } \\
\text { Time }\end{array}$ & $\begin{array}{l}\text { Previous } \\
\text { method }\end{array}$ & $\begin{array}{l}\text { Proposed } \\
\text { method }\end{array}$ \\
\hline 200 & $40.28(\mathrm{~s})$ & $2.62(\mathrm{~s})$ \\
\hline 400 & 91.23 & 6.03 \\
\hline 600 & 155.76 & 9.15 \\
\hline 800 & 206.97 & 12.65 \\
\hline 1000 & 266.86 & 16.96 \\
\hline
\end{tabular}




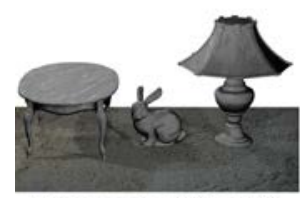

Simulation Time : 0

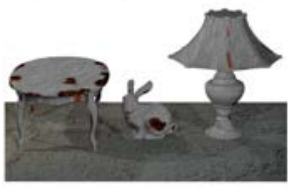

400

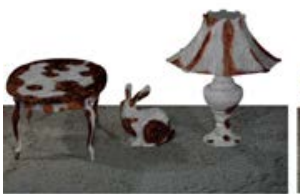

800

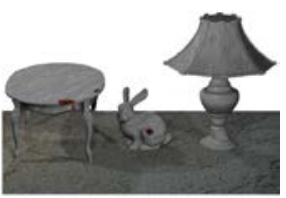

200

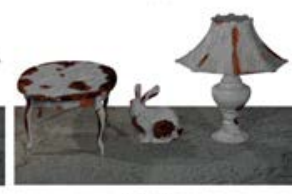

600

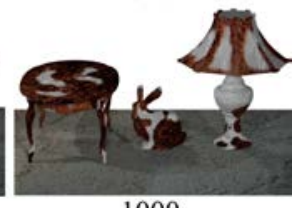

1000
Fig. 7: The simulation results of proposed methods to multi objects.

\section{Conclusion}

The proposed method, which considers the effects of peeling of a preservative coating and corrosion on an object's surface, reproduces a realistic agedeteriorated texture.

However, the proposed method cannot reproduce completely the deterioration phenomenon in texture spaces yet. In future, we intend to study accurate chemical corrosion expansion and the calculation of shape distortion by collision for more realistic reproduction of metal objects.

\section{References}

[1] N. Merillou, S. Merillou D. Ghazanfarpour, J. M. Dischler, E. Galin, "Simulating Atmospheric Pollution Weathering on Buildings," Proc. Int'l Central Europe Conf. Computer Graphics, Visualization and Computer Vision (WSCG 10), pp. 65-72, 2010.
[2] O. Clement, E. Paquette, “Adaptable aging factory for multiple objects and colorations," Computers \& Graphics 34, pp.460-467, 2010.

[3] N. Merillou, S. Merillou, E. Galin, D. Ghazanfarpour, "Simulating how salt decay ages buildings,” IEEE Computer Graphics and Applications, pp. 44-54, 2012.

[4] Y. -X. Chang, Z. -C. Shih, "Physically-Based Patination for Underground Objects," Comput. Graph. Forum 19, 3, pp. 109-117, 2000.

[5] M. M. Stack, S. M. Abdelrahman, B. D. Jana, “A new methodology for modeling erosion-corrosion regimes on real surfaces: Gliding down the galvanic series for a range of metalcorrosion systems," Wear 268, pp. 533-542, 2010.

[6] T. -T. Wong, W. -Y. Ng, P. -A. Heng, "A Geometry Dependent Texture Generation Framework for Simulating Surface Imperfections," Proceedings of the Eurographics Workshop on Rendering Techniques '97, pp.139-150, June 16-18, 1997.

[7] J. Lu, A. S. Georghiades, A. Glaser, H. Wu, L. -Y. Wu, B. Guo, J. Dorsey, H. Rushmeier, "Context-aware textures," ACM Transactions on Graphics (TOG), 26, 1, Article No. 3, 2007

[8] Y. -X. Chang, Z. -C. Shih, "The synthesis of rust in seawater," Visual Comput, 19, 1, 50-66, 2003.

[9] S. Merillou, J. -M Dischler, D. Ghazanfarpour, "Corrosion: Simulating and Rendering,” In Graphics interface, pp.167-174, June 07-09, 2001.

[10] Itoh, Y., Iwata, A., and Kainuma, S. , "Accelerated exposure tests of environmental durability for steels and the estimation of acceleration coefficient”, Journal of Structural Engineering, JSCE, Vol. 48A, pp.10211029, 2002. 